\title{
Entanglement oscillations in non-Markovian quantum channels
}

\author{
Sabrina Maniscalco, ,, S Stefano Olivares, 2, , \\ ${ }^{I}$ Department of Physics, University of Turku, FI-20014 Turku, Finland \\ ${ }^{2}$ Dipartimento di Fisica dell'Università di Milano, I-20133, Italia \\ ${ }^{3}$ Institute for Scientific Interchange Foundation, I-10133, Torino, Italia
}

(Dated: November 6, 2018)

\begin{abstract}
We study the non-Markovian dynamics of a two-mode bosonic system interacting with two uncorrelated thermal bosonic reservoirs. We present the solution to the exact microscopic Master equation in terms of the quantum characteristic function and study in details the dynamics of entanglement for bipartite Gaussian states. In particular, we analyze the effects of short-time system-reservoir correlations on the separability thresholds and show that the relevant parameter is the reservoir spectral density. If the frequencies of the involved modes are within the reservoir spectral density entanglement persists for a longer time than in a Markovian channel. On the other hand, when the reservoir spectrum is out of resonance short-time correlations lead to a faster decoherence and to the appearance of entanglement oscillations.

PACS numbers: 03.65.Yz, 03.67.Mn
\end{abstract}

The loss of coherence, or decoherence, within a quantum system is due to the interactions of the system with the surrounding environment. Decoherence processes cannot be ignored, for they are the main obstacle to the full realization of quantum information processing. The dynamics of open quantum systems, however, may be rather involved, mostly due to the complex structure of the environment interacting with the quantum system. Therefore, in order to describe the dynamics of the system of interest, some approximations are often made, leading to the derivation of a Master equation for the reduced density matrix. The most relevant approximations are the weak coupling or Born approximation [1, 2], assuming that the coupling between the system and the reservoir is small enough to justify a perturbative approach, and the Markov approximation [1, 2], which amounts to neglect shorttime correlations between the system and the reservoir. There are few cases, however, where an exact analytic description of the dynamics is possible. Two relevant examples are the quantum Brownian motion (QBM) [1, 2, 3, 4] and the case of a two-level atom interacting with a thermal reservoir with Lorentzian spectral density [1, 5].

Entanglement in continuous variable (CV) quantum channels has attracted much interest in recent years, due to potential improvement in the channel capacity [6]. A realistic analysis of $\mathrm{CV}$ channels must take into account decoherence and dissipation phenomena and, in fact, there has been an increasing interest in the description of noisy CV quantum channels [7]. Most of the theoretical descriptions, however, rely on Born and/or Markov approximations and only very recently some phenomenological models of non-Markovian quantum channels have been proposed [8, 9]. Non-Markovian effects are crucial, e.g., for high-speed quantum communication where the characteristic time scales become comparable with the reservoir correlation time. Moreover, when the sys-

\footnotetext{
*Electronic address: sabrina.maniscalco@utu.fi

${ }^{\dagger}$ Electronic address: stefano.olivares@mi.infn.it

${ }^{\ddagger}$ Electronic address: matteo.paris@ fisica.unimi.it
}

tem interacts with a structured reservoir, e.g., for quantum channels embedded in solid-state devices, memory effects are typically non negligible. In these cases the dynamics can be substantially different from the Markovian one.

In this communication we focus attention on the dynamics of a two-mode bosonic quantum system propagating in a noisy bosonic quantum channel, i.e., interacting with two uncorrelated bosonic thermal reservoir. Such a system may be seen as made by two quantum Brownian oscillators and therefore it is possible to exactly describe the system dynamics. Using this analogy, we generalize the single mode QBM solution to the bimodal case and analyze in details the entanglement dynamics of bipartite Gaussian state. We present an exact approach based on a microscopic model and, starting from the exact solution, we discuss in details the genuine non-Markovian effects on the entanglement dynamics for the relevant class of bipartite Gaussian states. In particular we analyze the effects of short-time system-reservoir correlations on the separability thresholds. The exact results are compared with those obtained within the Markovian approximation, and few relevant situations in which the system-reservoir correlations give rise, for short times, to entanglement oscillations are addressed. We stress that our approach describes the effects of quantum noise for two-mode quantum systems starting from a microscopic description of the system and the reservoir.

The exact Master equation describing a quantum harmonic oscillator interacting with a bosonic reservoir in thermal equilibrium has been derived for the first time in Ref. [4] and it is usually referred to as Hu-Paz-Zhang Master equation. The original derivation is based on the influence functional-path integral formalism though a simpler derivation based on the time-convolutionless projection operator technique [1] may be also used [see, e.g., Ref. [10]]. Assuming that the bimodal field interacts bilinearly with two identical uncorrelated bosonic thermal reservoirs the Master equation for the 
reduced density matrix $\varrho(t)$ of the field is given by

$$
\begin{aligned}
\dot{\varrho}(t)= & \sum_{k}\left\{\frac{1}{i \hbar}\left[H_{k}^{0}, \varrho(t)\right]-\Delta(t)\left[X_{k},\left[X_{k}, \varrho(t)\right]\right]\right. \\
& +\Pi(t)\left[X_{k},\left[P_{k}, \varrho(t)\right]\right]+\frac{i}{2} r(t)\left[X_{k}^{2}, \varrho(t)\right] \\
& \left.-i \gamma(t)\left[X_{k},\left\{P_{k}, \varrho\right\}\right]\right\},
\end{aligned}
$$

where

$$
X_{k}=\frac{1}{\sqrt{2}}\left(a_{k}+a_{k}^{\dagger}\right) \quad P_{k}=\frac{i}{\sqrt{2}}\left(a_{k}^{\dagger}-a_{k}\right)
$$

are the dimensionless quadrature operators, $\left[a_{k}, a_{k}^{\dagger}\right]=1$ ( $k=1,2)$ being the mode operators of the two oscillators, and $H_{k}^{0}=\hbar \omega_{0}\left(a_{k}^{\dagger} a_{k}+1 / 2\right)$. This Master equation, being exact, describes also the non-Markovian system-reservoir correlations due to the finite correlation time of the reservoir. In contrast to other non-Markovian dynamical models [11], Eq. (1) is local in time, i.e. it does not contain memory integrals. All the non-Markovian character of the system is contained in the time dependent coefficients, $\Delta(t), \Pi(t), r(t)$ and $\gamma(t)$, appearing in the Master equation. These coefficients depend only on the reservoir spectral density, i.e. on the microscopic effective coupling strength between the system oscillator and the oscillators of the reservoir. The coefficient $r(t)$ describes a time dependent frequency shift, $\gamma(t)$ is the damping coefficient, $\Delta(t)$ and $\Pi(t)$ are the normal and the anomalous diffusion coefficients, respectively [1, 3]. It is worth underlining that the Master equation given by Eq. (1), is valid for general forms of the reservoir spectral density $J(\omega)$ and any temperature $T$.

The solution of Eq. (1) can be obtained generalizing to twomodes the method of solution of the Hu-Paz-Zhang Master equation. Several approaches have been proposed in the literature to solve the Hu-Paz-Zhang Master equation [12, 13, 14]. The one developed in Refs. [13, 14] is based on the symmetrically ordered quantum characteristic function $(\mathrm{QCF}) \chi_{t}(\xi)$ at time $t$. Extending this approach to the case of a bimodal field, in the interaction picture with respect to $\sum_{k=1,2} H_{k}^{0}$ we obtain the following solution

$$
\chi_{t}(\boldsymbol{\Lambda})=\exp \left\{-\boldsymbol{\Lambda}^{T} \boldsymbol{A}_{t} \boldsymbol{\Lambda}\right\} \chi_{0}\left(e^{-\Gamma(t)} \boldsymbol{\Lambda}\right),
$$

where we indicate with $\chi_{0}(\boldsymbol{\Lambda})$ the characteristic function at $t=0$, and where $\boldsymbol{\Lambda}=\left(\mathrm{x}_{1}, \mathrm{y}_{1}, \mathrm{x}_{2}, \mathrm{y}_{2}\right)^{T},(\cdots)^{T}$ being the transposition operation. The bipartite QCF is defined as

$$
\chi(\boldsymbol{\Lambda})=\operatorname{Tr}\left[e^{\xi_{1} a_{1}^{\dagger}-\xi_{1}^{*} a_{1}} e^{\xi_{2} a_{2}^{\dagger}-\xi_{2}^{*} a_{2}} \varrho\right],
$$

with $\xi_{k}=\frac{1}{\sqrt{2}}\left(\mathrm{x}_{k}+i \mathrm{y}_{k}\right)$, and $k=1,2$. From the QCF one evaluates the moments of the field

$$
\begin{array}{r}
\left\langle a_{1}^{\dagger k} a_{2}^{\dagger l} a_{1}^{m} a_{2}^{n}\right\rangle=(-)^{m+n} \partial_{\xi_{1}}^{k} \partial_{\xi_{2}}^{l} \partial_{\xi_{1}^{*}}^{m} \partial_{\xi_{2}^{*}}^{n} e^{\frac{1}{2}\left(\left|\xi_{1}\right|^{2}+\left|\xi_{2}\right|^{2}\right)} \\
\times\left.\chi\left(\xi_{1}, \xi_{2}\right)\right|_{\xi_{1}=0, \xi_{2}=0},
\end{array}
$$

and, in turn, the time evolution of the covariance matrix, thus quantifying the entanglement between the two modes. In Eq. (2) the matrix $\boldsymbol{A}_{t}$ is given by [14]

$$
\boldsymbol{A}_{t}=e^{-\Gamma(t)} \int_{0}^{t} d s e^{\Gamma(s)} \boldsymbol{R}^{T}(t, s) \boldsymbol{M}(s) \boldsymbol{R}(t, s),
$$

where the matrix $\boldsymbol{R}(t, s)$ contains rapidly oscillating terms and $\boldsymbol{M}(t)$ is given by

$$
\boldsymbol{M}(t)=\frac{1}{2}\left(\begin{array}{cc}
2 \Delta(t) & -\Pi(t) \\
-\Pi(t) & 0
\end{array}\right) .
$$

It is worth recalling that the time dependent coefficients $\Delta(t)$, $\Pi(t)$ and $\gamma(t)$ depend only on the spectral density of the reservoir and can be expressed as power series in the systemreservoir coupling constant $\alpha$. Finally, $\Gamma(t)$ takes the form

$$
\Gamma(t)=2 \int_{0}^{t} d s \gamma(s) .
$$

Once the spectral characteristics of the environment and the initial state of the field have been specified, then Eq. (2) fully characterizes the dynamics of the system without approximations.

We now consider a specific example of interest for $\mathrm{CV}$ quantum information and communication, namely the case of a bipartite Gaussian state of the form

$$
\chi_{0}(\boldsymbol{\Lambda})=\exp \left\{-\frac{1}{2} \boldsymbol{\Lambda}^{T} \boldsymbol{\sigma}_{0} \boldsymbol{\Lambda}-i \boldsymbol{\Lambda}^{T} \overline{\boldsymbol{X}}_{\text {in }}\right\},
$$

where

$$
\sigma_{0}=\left(\begin{array}{l|l}
A & C \\
\hline C & B
\end{array}\right)
$$

with $\boldsymbol{A}=\operatorname{Diag}(a, a), \boldsymbol{B}=\operatorname{Diag}(b, b), a, b>0$, and $\boldsymbol{C}=\operatorname{Diag}\left(c_{1}, c_{2}\right)$ is the covariance matrix in the canonical form and $\overline{\boldsymbol{X}}_{\text {in }}=\operatorname{Tr}\left[\varrho(t)\left(X_{1}, P_{1}, X_{2}, P_{2}\right)^{T}\right]$ the vector of mean values. For optical bimodal fields the solution of the Master equation can be simplified invoking the secular approximation. It has been shown that, in this case, the time evolution depends only on the diffusion coefficient $\Delta(t)$ and on the dissipation coefficient $\gamma(t)[10,13,14]$. The expression for the QCF at time $t$ becomes

$$
\chi_{t}(\boldsymbol{\Lambda})=\exp \left\{-\frac{1}{2} \boldsymbol{\Lambda}^{T} \boldsymbol{\sigma}_{t} \boldsymbol{\Lambda}-i \boldsymbol{\Lambda}^{T} \overline{\boldsymbol{X}}_{\mathrm{t}}\right\}
$$

where the evolved mean value and covariance matrix are given by

$$
\begin{aligned}
\overline{\boldsymbol{X}}_{t} & =e^{-\Gamma(t) / 2}(R \oplus R)^{T} \overline{\boldsymbol{X}}_{\mathrm{in}}, \\
\boldsymbol{\sigma}_{t} & =e^{-\Gamma(t)}(R \oplus R)^{T} \boldsymbol{\sigma}_{0}(R \oplus R)+\frac{1}{2} \Delta_{\Gamma}(t) \mathbb{1},
\end{aligned}
$$

$\mathbb{1}$ being the $4 \times 4$ identity matrix and $R$ the rotation matrix

$$
R=\left(\begin{array}{cc}
\cos \omega_{0} t & \sin \omega_{0} t \\
-\sin \omega_{0} t & \cos \omega_{0} t
\end{array}\right)
$$


Finally, the time dependent coefficient $\Delta_{\Gamma}(t)$ appearing in Eq. (11) is given by

$$
\Delta_{\Gamma}(t)=e^{-\Gamma(t)} \int_{0}^{t} d s e^{\Gamma(s)} \Delta(s)
$$

The map expressed by Eqs. (10) and (11) is Gaussian, i.e. it maintains the Gaussian character of the input state. As a consequence, separability after interactions may be checked by positivity of the partially transposed density matrix (PPT condition) [16, 17], which, in terms of the covariance matrix, reads as follows

$$
\boldsymbol{\sigma}_{t}+\frac{i}{2} \boldsymbol{\Omega} \geq 0,
$$

where $\boldsymbol{\Omega}=\boldsymbol{\omega} \oplus \boldsymbol{\omega}^{T}$, with

$$
\boldsymbol{\omega}=\left(\begin{array}{cc}
0 & 1 \\
-1 & 0
\end{array}\right)
$$

Explicitly, for the evolved state of Eq. (9), the PPT condition may be written as

$$
\begin{aligned}
& {\left[(a+b) e^{-\Gamma(t)}+\Delta_{\Gamma}(t)\right]^{2}} \\
& \quad-e^{-\Gamma(t)}\left\{\left[(a-b)^{2}+2\left(c_{1}^{2}+c_{2}^{2}\right)\right] e^{-\Gamma(t)}\right. \\
& \left.-2 \sqrt{(a-b)^{2}+\left(c_{1}-c_{2}\right)^{2}+\left(c_{1}^{2}-c_{2}^{2}\right)^{2} e^{-2 \Gamma(t)}}\right\} \geq 1 .
\end{aligned}
$$

In the case of a twin-beam (TWB) state of radiation, i.e.,

$$
a=b=\frac{1}{2} \cosh (2 r) \quad c_{1}=-c_{2}=\frac{1}{2} \sinh (2 r),
$$

$r>0$, the last inequality reduces to [18]

$$
S(t)=e^{-2 r} e^{-\Gamma(t)}+\Delta_{\Gamma}(t)-1 \geq 0 .
$$

Notice that, since in the case of a Gaussian state there is no bound entanglement, the sign of $S(t)$ is strictly connected to the separability of the state: when $S(t) \geq 0$ the state is separable and, for this reason, $S(t)$ is called separability function. At $t=0, \Gamma(0)=\Delta_{\Gamma}(0)=0$, hence $S(0)=e^{-2 r}-1$, i.e. the initial state is an entangled state. As time passes, due to the interaction with the external environment, the entanglement between the modes deteriorates and the separability function $S(t)$ vanishes. The time $t_{\mathrm{s}}$ at which $S\left(t_{\mathrm{s}}\right)=0$, i.e. when the entanglement between the two modes is lost, is referred to as the separability threshold or separability time. Of course, $t_{\mathrm{s}}$ is a function of both the system and the reservoir parameters. In the Markovian approximation, for example, $t_{\mathrm{s}}$ has been shown to become infinite for a zero $T$ reservoir [19].

In order to study in more detail the entanglement dynamics in a non-Markovian channel, we need to specify the spectral density of the reservoir. We focus on the Ohmic reservoir with Lorentz-Drude regularization, whose spectral density is [2]

$$
J(\omega)=\frac{\omega}{\pi} \frac{\omega_{c}^{2}}{\omega_{c}^{2}+\omega^{2}},
$$

where $\omega_{c}$ is the cutoff frequency. This is one of the most studied models of bosonic environments since it leads to a friction force proportional to velocity, which is typical of dissipative systems in several physical contexts. In this model, the reservoir correlation time is given by the inverse of the cutoff frequency, i.e. $\tau_{R}=1 / \omega_{c}$. For times $t \leq \tau_{R}$ the system dynamics cannot be approximated by the Markovian dynamics, as we will show in the following. The expressions for the relevant time dependent coefficients, up to second order in the system-reservoir coupling constant, are given by [4, 15]

$$
\begin{aligned}
& \Delta(t)=\int_{0}^{t} d \tau \kappa(\tau) \cos \left(\omega_{0} \tau\right) \\
& \gamma(t)=\int_{0}^{t} d \tau \mu(\tau) \sin \left(\omega_{0} \tau\right)
\end{aligned}
$$

with

$$
\begin{aligned}
& \kappa(\tau)=\alpha^{2} \int_{0}^{\infty} d \omega J(\omega) \operatorname{coth}\left[\hbar \omega / 2 k_{B} T\right] \cos (\omega \tau) \\
& \mu(\tau)=\alpha^{2} \int_{0}^{\infty} d \omega J(\omega) \sin (\omega \tau)
\end{aligned}
$$

being the noise and the dissipation kernels, respectively [1]. We note that, for high reservoir temperatures $T, \Delta(t) \gg \gamma(t)$. Inserting the expression of $J(\omega)$ into Eqs. (18) and (19) one gets analytic expressions for both of the coefficients for the high $T$ case. For generic temperatures only $\gamma(t)$, which is independent of temperature, has a simple analytic form, while the time integral in $\Delta(t)$ has to be calculated numerically [see, e.g., Ref. [15] for the analytic expression of the diffusion and dissipation coefficients]. We begin our analysis by noting that, for times $t \gg \tau_{R}$, the coefficients $\Delta(t)$ and $\gamma(t)$ can be approximated by their Markovian stationary values $\Delta_{M}=\Delta(t \rightarrow \infty)$ and $\gamma_{M}=\gamma(t \rightarrow \infty)$ [see Appendix B of Ref. [15]]. Eqs. (6) and (13), hence, become

$$
\begin{aligned}
\Gamma(t) & =\gamma_{M} t \\
\Delta_{\Gamma}(t) & =\left[2 N\left(\omega_{0}\right)+1\right]\left(1-e^{-\gamma_{M} t}\right)
\end{aligned}
$$

where $N\left(\omega_{0}\right)=\left(e^{\hbar \omega_{0} / k_{B} T}-1\right)^{-1}$ is the average number of thermal photons. Inserting Eqs. (22) and (23) into Eq. (17), one can easily see that the separability time coincide with that predicted by the Markovian theory [18, 19], i.e

$$
t_{s}=\frac{1}{\gamma_{M}} \log \left(1+\frac{1-e^{-2 r}}{2 N\left(\omega_{0}\right)}\right) .
$$

Notice that, as mentioned above, if $N\left(\omega_{0}\right) \rightarrow 0$ then $t_{\mathrm{s}} \rightarrow \infty$.

We now look at the deviation from the Markovian value of the separability time for times $t \leq \tau_{R}$. In this time interval the expression for the separability condition $17 \mathrm{~b}$ reduces to

$$
S(t) \simeq e^{-2 r}\left(1-\int_{0}^{t} d s \gamma(s)\right)+\int_{0}^{t} d s \Delta(s)-1 \geq 0 .
$$


We consider the high $T$ case. Using the analytic expressions of $\Delta(t)$ and $\gamma(t)$, we get

$$
\begin{array}{r}
S(\tau)=\alpha^{2} \frac{k_{B} T}{\hbar \omega_{c}} \frac{x^{2}}{1+x^{2}}\left\{\tau-\frac{x^{2}-1}{x^{2}+1}\left[1-e^{-\tau} \cos (\tau / x)\right]\right. \\
\left.-2 \frac{x}{x^{2}+1} e^{-\tau} \sin (\tau / x)\right\}+e^{-2 r}-1,
\end{array}
$$

with $\tau=\omega_{c} t$, and $x=\omega_{c} / \omega_{0}$. In Fig. 1 we report the separability function $S(t)$, and we compare it with the corresponding Markovian approximation

$$
S_{M}(\tau)=\frac{\tau}{2} \frac{\alpha^{2} x}{1+x^{2}}\left(1+2 x \frac{k_{B} T}{\hbar \omega_{c}}\right)+e^{-2 r}-1 .
$$
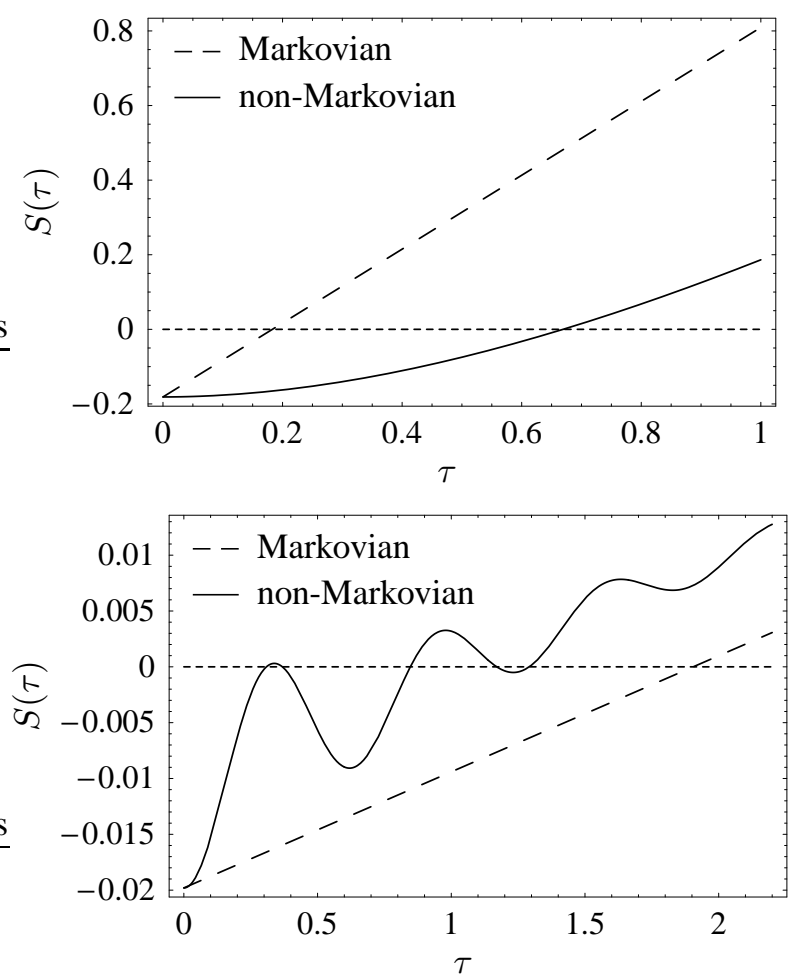

FIG. 1: Separability function $S(\tau)$ for twin-beam state in Markovian and non-Markovian channels for a high $T$ reservoir. Plots refer to parameters $k_{B} T / \hbar \omega_{c}=100, \alpha^{2}=0.01, r=0.1$, and (Top): $x=10$; (Bottom): $x=0.01$. The top plot refers to a situation in which the frequencies of the involved modes overlap with the reservoir spectral density $(x \gg 1)$. Here, the more accurate non-Markovian estimation predicts that the entanglement persists for a longer time. This conclusion holds for any value of the reservoir temperature, provided the high $T$ condition is satisfied, and as long as $x \gg 1$. The bottom plot refers to a situation with the reservoir spectrum out of resonance with respect to the mode frequencies, i.e. when $x \ll 1$. Here, the environment causes a faster loss of entanglement compared to the Markovian prediction, as well as to the appearance of entanglement oscillations.
The case considered in the top plot corresponds to a situation in which the frequencies of the involved modes overlap with the reservoir spectral density $(x \gg 1)$. The Markovian prediction for the separability time, in this case, gives a smaller value than the one predicted by our non-Markovian theory. More precisely we have $\tau_{\mathrm{s}} \simeq 0.18 \leq \tau_{\mathrm{s}} \simeq 0.65$. Therefore, the more accurate non-Markovian estimation predicts that the entanglement persists for a longer time. It is easy to show that this conclusion holds for any value of the reservoir temperature, provided the high $T$ condition is satisfied, and as long as $x \gg 1$.

The situation changes for the case in which the reservoir spectrum is "out of resonance" or "detuned" with respect to the frequency of the TWB, i.e. when $x \ll 1$. As shown in Fig. 1 (bottom plot), in this case the environment causes a faster loss of entanglement compared to the Markovian prediction. A careful analysis of Eq. (17) shows that this feature can be traced back to the short time behavior of the diffusion coefficient $\Delta(t)$, describing environment induced decoherence [4]. For $x \gg 1$, indeed, $\Delta(t) \geq \Delta_{M}$, with $\Delta_{M}=\Delta(t \rightarrow \infty)$ the Markovian value. Therefore, in this case, the system experiences a weaker decoherence than the one predicted by the Markovian approximation and, as a consequence, the entanglement between the modes persists longer. On the contrary, for $x \ll 1, \Delta(t)$ quickly assumes values higher than $\Delta_{M}$. The system hence is subjected to a stronger initial decoherence which quickly destroys the quantum entanglement between the two modes. In Fig. 1 we see that, for $x \ll 1$, the separability function displays entanglement oscillations, a typical non-Markovian feature. Similar oscillations have been extensively studied for the case of the one-mode harmonic quantum Brownian model [20]. It is worth noting, however, that here we look at quantum correlations between two modes whose dynamics cannot be directly deduced from the dissipative dynamics of a single mode bosonic quantum channel.

In conclusion, we have analyzed the dynamics of bipartite Gaussian states in a non-Markovian noisy channel. We found that when the mode frequencies are within the reservoir spectral density entanglement persists for a longer time than in a Markovian channel. On the other hand, when the reservoir spectrum is out of resonance than short-time correlations lead to a faster decoherence and to the appearance of entanglement oscillations. Our analysis indicates that non Markovian features of system-reservoir interaction may be relevant in quantum information processing and should be taken into account in the design of any quantum communication channel.

This work has been supported by MIUR through the project PRIN-2005024254-002. S.M. acknowledges financial support from the Academy of Finland (projects 108699,115682) and from the Magnus Ehrnrooth Foundation. 
[1] H.-P. Breuer and F. Petruccione, The Theory of Open Quantum systems (Oxford University Press, 2002).

[2] U. Weiss, Quantum Dissipative Systems, 2nd edition (World Scientific Publishing, Singapore, 1999).

[3] W. H. Zurek, Rev. Mod. Phys. 75, 715 (2003).

[4] B. L. Hu, J. P. Paz, and Y. Zhang, Phys. Rev. D 452843 (1992).

[5] B. M. Garraway, Phys. Rev. A 55, 2290 (1997).

[6] A. S. Holevo and R. Werner, Phys. Rev. A 63, 032312 (2001).

[7] A. Serafini et al., J. Opt. B 7, R19-R36 (2005).

[8] M. Ban J. Phys. A 39. 1927 (2006); Phys. Lett. A 359, 402 (2006).

[9] H. McAneney et al., J. Mod. Opt. 52, 935 (2005).

[10] F. Intravaia, S. Maniscalco, and A. Messina, Eur. Phys. J. B 32, 97 (2003).

[11] S.M. Barnett and S. Stenholm, Phys. Rev. A 64, 033808 (2001).

[12] J. J. Halliwell and T. Yu Phys. Rev. D 53, 2012 (1996); R. Karrlein and H. Grabert Phys. Rev. E 55, 153 (1997); G. W. Ford and R. F. O’Connell, Phys. Rev. D 64, 105020 (2001).

[13] F. Intravaia, S. Maniscalco, and A. Messina, Phys. Rev. A 67, 042108 (2003).

[14] S. Maniscalco, F. Intravia, J. Piilo, and A. Messina, J. Opt. B 6, S98 (2004).

[15] S. Maniscalco et al., Phys. Rev. A 70, 032113 (2004).

[16] A. Peres, Phys. Rev. Lett. 77, 1413 (1996).

[17] R. Simon, Phys. Rev. Lett. 842726 (2000).

[18] J. S. Prauzner-Bechcicki, J. Phys. A 37, L173 (2004).

[19] S. Olivares, M. G. A. Paris, and A. R. Rossi, Phys. Lett. A 319, 32 (2003); A. R. Rossi, S. Olivares, and M. G. A. Paris, J. Mod. Opt. 51, 1057 (2004).

[20] F. Intravaia, S. Maniscalco, J. Piilo, and A. Messina, Phys. Lett. A 308, 6 (2003); S. Maniscalco, J. Piilo, F. Intravaia, F. Petruccione, and A. Messina, Phys. Rev. A 69, 052101 (2004); S. Maniscalco, J. Opt. B 7, R1 (2005). 